Audiology

Neurotology
Audiol Neurotol 2016;21:333-345

DOI: $10.1159 / 000454666$
Received: May 11, 2016

Accepted after revision: November 22, 2016

Published online: January 5, 2017

\title{
A Retrospective Multicentre Cohort Review of Patient Characteristics and Surgical Aspects versus the Long-Term Outcomes for Recipients of a Fully Implantable Active Middle Ear Implant
}

\author{
Philippe P. Lefebvre ${ }^{a} \quad$ Javier Gisbert ${ }^{b}$ Domenico Cuda ${ }^{c}$ Stéphane Tringalic, ${ }^{c}$ \\ Arnaud Deveze ${ }^{e}$ \\ ${ }^{a}$ Department of Otorhinolaryngology, CHU de Liège, Liège, Belgium; ${ }^{b}$ Department of ENT, Hospital de la \\ Ribera, Alzira, Spain; ' Otorhinolaryngology Unit, "Guglielmo de Saliceto" Hospital, Piacenza, Italy; ${ }^{\text {d Department }}$ \\ of Otolaryngology-Head and Neck Surgery, Hospices Civils de Lyon, Lyon-Sud Hospital, Pierre-Bénite, and \\ e Department of Otolaryngology and Skull Base Surgery, Ramsay-Générale de Santé, Hospital Clairval, Marseille, \\ France
}

\begin{abstract}
Keywords
Active middle ear implant · Feedback · Patient satisfaction · Cochlear Carina · Otologics Carina · Implantable microphone · Surgery
\end{abstract}

\begin{abstract}
Objective: To summarise treatment outcomes compared to surgical and patient variables for a multicentre recipient cohort using a fully implantable active middle ear implant for hearing impairment. To describe the authors' preferred surgical technique to determine microphone placement. Study Design: Multicentre retrospective, observational survey. Setting: Five tertiary referral centres. Patients: Carina recipients ( 66 ears, 62 subjects) using the current Cochlear ${ }^{\circledR}$ Carina ${ }^{\circledR}$ System or the legacy device, the Otologics ${ }^{\circledR}$ Fully Implantable Middle Ear, with a T2 transducer. Methods: Patient file review and routine clinical review. Patient outcomes assessed were satisfaction, daily use and feedback reports at the first fitting and $\geq 12$ months after implantation. Descriptive and statistical analysis of correlations of variables and
\end{abstract}

their influence on outcomes was performed. Independently reported preferred methods for microphone placement are collectively summarised. Results: The average implant experience was 3.5 years. Satisfaction increased significantly over time $(p<0.05)$. No correlation with covariates examined was observed. Feedback significantly decreased over time, showing a significant correlation with microphone location, primary motivation, gender, age at implantation, and contralateral hearing aid use $(p<0.05)$. Patient satisfaction was inversely correlated with reports of system feedback $(p<0.05)$. The implantable microphone was most commonly on the posterior inferior mastoid line, in 42/66 (65\%) cases, correlating with less likelihood for feedback and consistent with author surgical preference. Conclusion: Carina recipients in this study present as satisfied consistent daily users with very few reports of persistent feedback. As microphone location is an influencing factor, a careful surgical consideration of microphone placement is required. The authors prefer a posterior inferior mastoid line position whenever possible.

(c) 2017 The Author(s)

Published by S. Karger AG, Basel

\begin{tabular}{ll}
\hline KARGER & $\begin{array}{l}\text { ( ) } 2017 \text { The Author(s) } \\
\text { Published by S. Karger AG, Basel }\end{array}$ \\
& Openger \\
E-Mail karger@karger.com & apesess \\
www.karger.com/aud & This article is licensed under the Creative Commons Attribution- \\
& NonCommercial-NoDerivatives 4.0 International License (CC BY- \\
& NC-ND) (http://www.karger.com/Services/OpenAccessLicense). \\
& Usage and distribution for commercial purposes as well as any dis- \\
tribution of modified material requires written permission.
\end{tabular}




\section{Introduction}

The use of an internally implantable microphone of a fully implantable active middle ear implant (AMEI) will influence the acoustic performance of the system, the daily users' physical comfort and the perception of sound quality and reception of desired external sound sources and undesired internal noises [Bruschini et al., 2010; Kam et al., 2013; Zwartenkot et al., 2013; Bittencourt et al., 2014; Pulcherio et al., 2014; Savas et al., 2016; Bruschini et al., 2016]. While the surgical considerations for placement of semi-implantable and fully implantable AMEIs remain largely the same, special attention is needed for the implantable microphone component. In addition to the technical aspects of identifying the most appropriate location for the placement, consideration of patient-specific anatomy, physical make-up and lifestyle is required to ensure optimal sound quality and wearer comfort.

Surgically, a few factors concerning microphone placement include the anatomical localization of the desired microphone location versus the dimensions of the microphone body, the ability to secure the microphone and the avoidance of unnecessary migration. Concurrently, the outcome should enable wearer comfort during daily activities and allow for tolerances of the body tissue layers with reference to the potential internal and external forces over the implanted site. Audiologically, it is important to consider the logical location of sound reception in the human body to facilitate sound awareness and sound reception, in particular, yet not exclusively, for conversational speech, the need to reduce the likelihood of any undesired amplification of body noises, and, last but not least, the need to reduce the likelihood of system feedback.

Carina system feedback typically manifests as a highpitched "squealing" tone reported by the recipients and clinically observed as reported previously [Jenkins et al., 2007; Bruschini et al., 2010]. The phenomenon of feedback is an oscillation caused by the hearing system's output being detected by its input, which varies with the feedback conditions and is distinct in nature from other artefacts such as reverberation, noise or distortion [Egolf et al., 1989]. The feedback loop created from a Carina system is similar to that observed for users of conventional amplification, with the exception that the feedback loop is transmitting to the microphone implanted under the skin.

To date, published guidelines on the identification of the optimal site for microphone placement, the surgical procedure and the impact upon outcomes have not been reported in detail, referring to "recommendations by the manufacturer" [Lefebvre et al., 2009; Martin et al., 2009; Bruschini et al., 2016]. Description of the microphone placement has been explored in a cohort of 32 ears as in the mastoid area with variations in the method used to place the microphone as either in "a subcutaneous pocket formed between the skin and the sternocleidomastoid muscle near the mastoid tip" for the majority of cases or as "fixed over the mastoid surface close to the mastoid tip" [Bruschini et al., 2016]. Clinically, they observed no influence of the microphone location upon system performance with particular mention of no impact upon the incidence of feedback which occurred rarely.

The only other fully implantable hearing implant including an implantable microphone, introduced on the market, was the Implex middle ear implant (Implex American Hearing Systems) in which the microphone was placed deeply in the external ear canal, close to the eardrum [Haynes et al., 2009]. This system was subsequently withdrawn in the 1990s due to technical difficulties and reduced long-term clinical benefits [Zenner and Leysieffer, 2001]. As the external ear canal is a biologically active dynamic environment and clinical experience would suggest that with the increased risk of feedback and potential for extrusion of implanted bodies, it should be avoided as a location for implantable microphones.

The main purpose of this study was to collect and summarise the authors' experiences with retrospectively treated Carina recipients, summarising the patient characteristics, surgical aspects including microphone placement, and to determine the influence of these variables upon patient satisfaction and reports of system feedback longitudinally. A secondary aim was to provide the consolidated co-author opinion on the preferred location for the microphone based on the collective clinical experience.

\section{Methods}

Clinical opinions for the optimal microphone placement were gathered from the 5 surgeons and co-authors of this report. Each author has several years of implant experience with the fully implantable AMEI (i.e. Otologics ${ }^{\circledR}$ Carina ${ }^{\circledR}$ followed by the Cochle$\operatorname{ar}^{\circledR}$ Carina ${ }^{\circledR}$ more recently). The authors of this paper were approached by mail for voluntary participation in the study. They were asked to independently describe their preferred surgical approach for microphone placement, based on their surgical experience, in an open-ended, unrestricted written format. The only guidelines provided for their surgical summary suggested inclusion of the following steps: evaluation, identification of the desired location and fixation for the implantable microphone. A collective summary of the individual preferences and recommendations of 
all 5 authors was prepared and shared for their approval and inclusion in the paper (see Appendix).

In parallel to compare the preferred surgical approach to their clinical practice for microphone placement, the authors were asked to perform a hospital file review and record patient demographics, surgical methods, and patient outcomes recorded at routine clinic visits for retrospectively implanted Carina recipients via an observational survey. Each surgeon was asked to put the appropriate data into a designated Excel template for each Carina recipient who was using the current version of the T2 actuator (i.e. Cochlear Carina and the legacy implant system Otologics Carina, also known as MET 7000) implanted from June 2010 to June 2015.

For purposes of our evaluation and analysis, only the long-term data for users of the Carina system including the T2 transducer design were targeted for survey and report. The aim was to reduce the influence of an already identified variable upon patient outcomes, i.e. earlier discontinued transducer configurations. The decision to exclude reports on cases using the earlier and superseded T1 transducer component, at the follow-up evaluation interval, was based on the variable design differences between the current $\mathrm{T} 2$ and T1 transducer versions, which display divergent transfer functions and resonant frequency characteristics that have inherently different influences upon the subsequent necessity to compensate for feedback occurring in situ.

Individual patient demographics were collected for age at implantation, gender, degree of hearing loss (pure-tone average, PTA, for air and bone conduction in each ear for $0.5,1.0,2.0$, and $4.0 \mathrm{kHz}$ ), hearing loss type in each ear (mixed/conductive/sensorineural), pre-implantation hearing aid (HA) usage in each ear, HA usage in the contralateral ear after implantation, average daily usage of the Carina system, ear canal direction and size, as well as patient motivations for implantation. Individual recipient and ear data collected are summarised via descriptive analysis.

Surgical details requested for each individual included method of microphone fixation, actuator in use at the first fitting and follow-up, location of actuator coupling, method of actuator site preparation, implant system brand and date of implantation. Description of the microphone location used for each recipient included was guided with reference to a graphical image of the head with 5 possible sites, i.e. from A to $\mathrm{E}$, which was provided to the surgeons for their responses (Fig. 1).

Longitudinal patient outcomes requested included system feedback at first switch-on and at $\geq 12$ months after implantation, permitting binary responses of "no" or "yes," and patient satisfaction at the same assessment intervals, permitting ordinal responses scored on a 6-point scale where 0 indicates "cannot judge" to 5 that indicates "very satisfied." Additionally for all recipients, consistency of use was evaluated by recording hours of device use per day for each individual. For recipients experiencing feedback, a further rating of the perceived degree of how bothersome the feedback experienced was requested via an analogue scale from 0 to 10 where 0 represents "not at all" and 10 is "very bothersome."

The Carina recipient cohort data provided by 3 of the 5 authors are summarised in this report. The remaining 2 authors had insufficient time to perform the requested individual patient file searches in their respective clinics.

\section{Statistical Analysis}

Frequency analysis of the collective responses for all surgical and patient variables examined and patient outcome ratings was

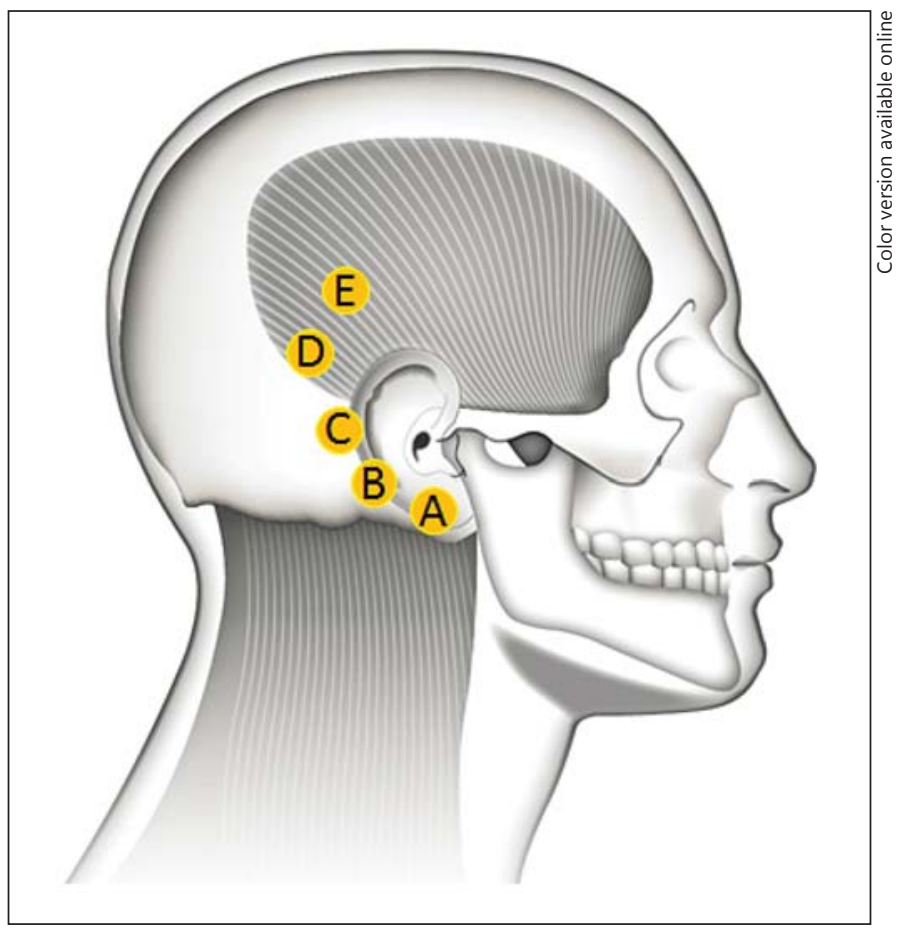

Fig. 1. Microphone location options provided for survey. A, mastoid tip; B, posterior inferior mastoid line; C, posterior mastoid; D, posterior; $\mathrm{E}$, posterior superior. Summary of reported microphone locations for 66 ears: $A=15 \%, B=65 \%, C=11 \%, D=9 \%$, and $\mathrm{E}=0 \%$, where position $\mathrm{A}$ is significantly linked to system feedback.

performed to provide a description of the responses provided. Mean and standard deviations were calculated and reported where applicable for continuous variables. Statistical analysis was performed to determine the correlation of surgical and patient demographic variables examined upon the main patient outcomes reported for the cohort $(n=62)$ with the level of significance set at $p<0.05$. For simplicity, bilateral user data $(n=4)$ were included for analysis of correlations with patient outcomes, with reference to the characteristics of the first implanted ear. Variables examined for possible influence upon patient outcomes were divided into categorical and continuous and presented accordingly. To determine the association between each categorical variable and the presence or absence of "system feedback" at the first fitting and $\geq 12$ months after implantation, a Fisher exact test was used. Logistic regression was applied for the remaining continuous variables. The McNemar test was used to determine changes over time in reports of feedback between the 2 intervals. To determine the association between each categorical variable and "patient satisfaction," ordinal regression was undertaken. Furthermore the potential relationship between the main outcomes, system feedback and patient satisfaction, was examined at each assessment interval, adopting $p<0.05$ as indicating a significant influence between the outcomes. Data analysis was performed using the downloadable statistical package of the R Core Team [2016]. Analysis of the collected data was implemented by an external consultant statistician at the Macquarie University, Sydney, Australia. 
Table 1. Patient demographics for continuous variables: correlation of covariates with reports of feedback over time

\begin{tabular}{|c|c|c|c|c|c|c|c|}
\hline & \multicolumn{3}{|c|}{ Feedback at first fitting } & \multicolumn{3}{|c|}{ Feedback at $>12$ months FU } & Total $(n=62)$ \\
\hline Duration of hearing loss, years & $20.6(11.3)$ & $22.3(10.1)$ & 0.602 & $21.4(11.6)$ & $21.5(8.1)$ & 0.984 & $21(11)[0.5-59]$ \\
\hline Time since implantation, years & $3.5(2.3)$ & $3.5(0.9)$ & 0.944 & $3.5(2.1)$ & $3.6(1.1)$ & 0.801 & $3.5(1.8)$ \\
\hline Time since last assessment, years & $0.7(0.7)$ & $1.0(0.5)$ & 0.151 & $0.8(0.7)$ & $0.8(0.4)$ & 0.846 & $0.8(0.6)$ \\
\hline HL degree ipsilateral ${ }^{1}$ (PTA-AC) & $64.7(12.6)$ & $68.3(12.9)$ & 0.296 & $64.8(12.1)$ & $70.5(14.4)$ & 0.212 & $66(13)[36-91]$ \\
\hline
\end{tabular}

Results are expressed as means with SD in parentheses or ranges in square brackets. $p$ values of significance are italicised. FU, follow-up; HL, hearing loss; PTA, pure-tone average for 0.5, 1.0, 2.0, and $4.0 \mathrm{kHz}$; AC, air conduction. ${ }^{1}$ The first implanted ear is designated as ipsilateral for 4 bilateral users for correlations.

\section{Results}

\section{Carina Recipient Demographics}

Data were provided for 62 recipients $(66$ ears including 4 of bilateral users) from 3 implant author sites. The summary cohort data for continuous variables are provided in Table 1.

The mean age at implantation is 39 years with a median of 40 . The duration of hearing loss prior to first implantation tended to be relatively long, on average over 2 decades, ranging from half a year to 59 years. At the time of data extract, the time since implant was 3.5 years (median 3.3, SD 1.8). Recipients included had been routinely reviewed at the clinic within the last 10 months on average. On average, the ipsilateral ear displayed a moderateto-severe degree of hearing loss with a PTA of $66 \mathrm{~dB} \mathrm{HL}$, ranging from mild to profound. The contralateral ear also displayed a moderate-to-severe hearing loss on average for the PTA, ranging from normal in 1 case to profound in others. Although not displayed, further examination of interaural asymmetry for air conduction PTA suggests a clinically significant difference between the ears when defined as $>15 \mathrm{~dB}$ difference for $14 / 62$ cases, with 6 implanted in the poorer ear and 8 in the better ear. Significant interaural asymmetry for bone conduction PTA was noted in 4 cases with 3 implanted in the poorer ear and 1 in the better ear.

Table 2 displays patient demographics for categorical variables examined for 62 individuals with 66 ears. The majority of recipients, $60 \%$, are male. The majority of cases (87-89\%) displayed a sensorineural hearing loss in both ears. The majority of cases, $79 \%$, wore an HA in the implanted ear, and $70 \%$ wore an HA both before and after implantation in the contralateral ear. Further examination of HA use shows that $71 \%$ (44/62) were bilateral HA users, approximately $20 \%$ wore no $\mathrm{HA}$, while $9 \%$ wore a unilateral HA only, 1 using it on the contralateral side. Various aetiologies (not displayed) were represented within the cohort; the most frequent was familial deafness for $37 / 66$ (56\%), followed by ototoxic drugs (11\%), and unknown in $12 \%$. The primary motivation for patients was work-related hearing needs for $52 \%$ and cosmetic reasons for $27 \%$. Two percent were motivated by not being able to wear an HA in the implanted ear and $19 \%$ by lifestyle issues. Ear canal demographics as visually judged by the surgeon are also presented in Table 2.

\section{Longitudinal Patient Outcomes}

The level of patient satisfaction reported at the first fitting and the follow-up intervals is illustrated in Figure 2. Very few recipients were reported as dissatisfied at either interval, most were reported as satisfied or very satisfied at the first fitting and the follow-up (91.9 and 95.2\%, respectively). In general, at $\geq 12$ months of follow-up, recipients were considered more satisfied than at the first fitting. Collapsing the satisfaction rating categories into very dissatisfied, dissatisfied and cannot judge (i.e. in view of the small number of responses across them) permitted statistical analysis via the McNemar test, which suggested a highly significant $(p<0.006)$ change in response categories. A higher number of recipients was rated as more satisfied at $\geq 12$ months after implantation than at the first fitting. On average, recipients reported wearing their system on a daily basis for $15 \mathrm{~h}$ (SD 2.6; range 1-18). Only 2 recipients were observed to wear their device for less than $10 \mathrm{~h} /$ day.

Reports of feedback are represented at the first fitting and follow-up assessment for the study cohort in Figure 3. At the first fitting, feedback was reported for $37 \%$ of recipients (23/62, 66 ears), which decreased signifi- 
Table 2. Patient demographics for categorical variables: correlation of covariates with reports of feedback over time

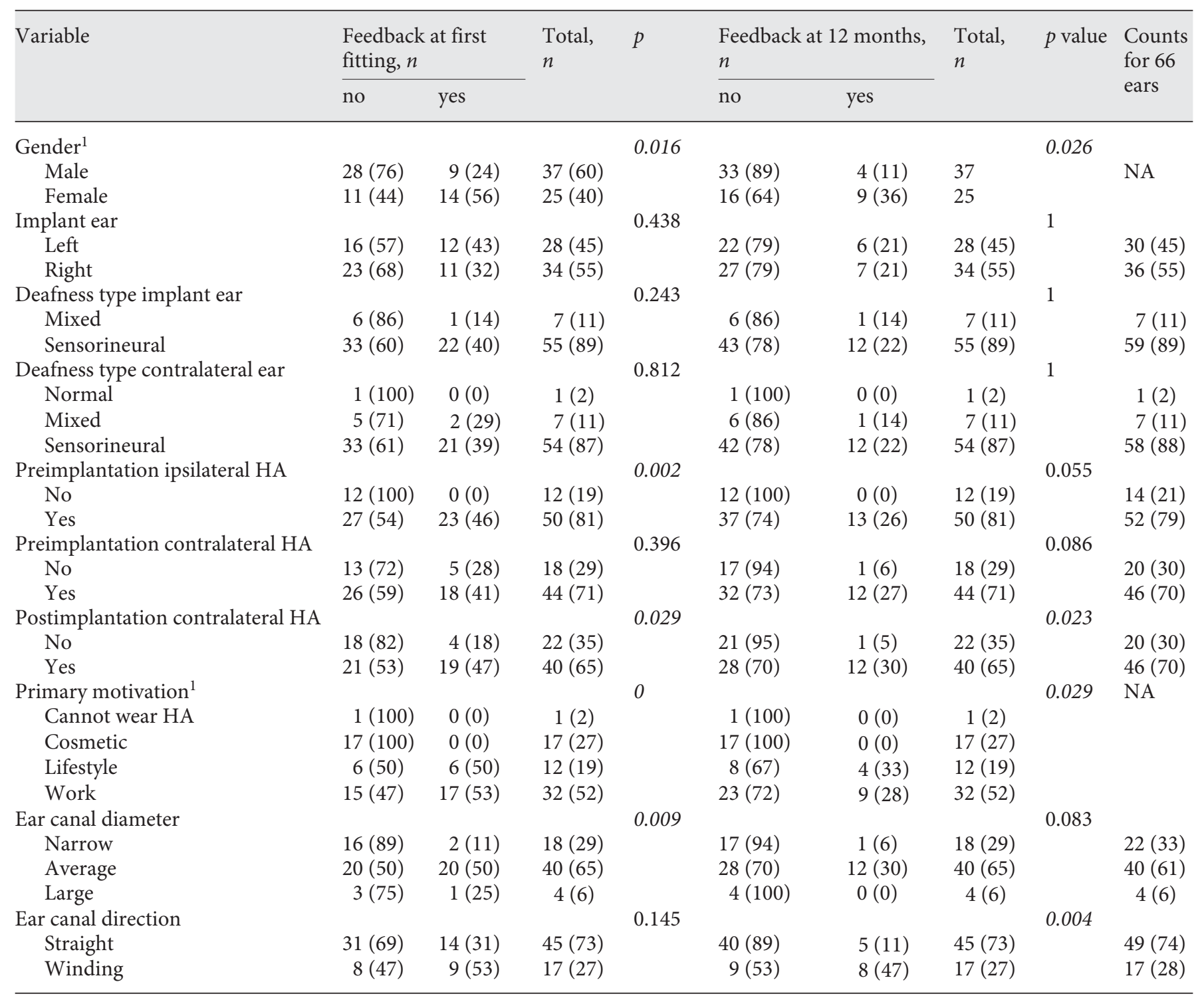

Figures in parentheses are percentages. $p$ values of significance are italicised. Ipsilateral ear is designated as the first implanted ear for 4 bilateral users. HA, hearing aid. ${ }^{1}$ Variables refer to responses from 62 subjects only.

cantly to approximately $1 / 5$ recipients $(13 / 62)$ at the $\geq 12$ months of follow-up interval $(p<0.0001)$. The "bothersome" rating of the tinnitus was requested for all recipients, regardless of their report of system feedback or not. A "bothersome" rating was recorded for $42 / 62$ recipients at their $\geq 12$-month assessment, with a "not at all bothersome" rating recorded for $22 / 42$ (52\%), a rating of $1-5$ for $16 / 42(38 \%)$ and $6-7$ for $2 / 42(5 \%)$, and no users had a rating of $8-9$. The remaining 2 users rated feedback as very bothersome, i.e. 10. Further examination revealed that both cases with very bothersome feedback were the only inconsistent users identified above, wearing their device for 1 and $3 \mathrm{~h}$ daily.

\section{Patient Variables versus Patient Outcomes}

Patient satisfaction did not correlate with any of the patient variables considered. In contrast as shown in Tables 1 and 2, for the same selected variables, a correlation was observed for several items with system feedback over times. In summary, remaining relative stable over time, 


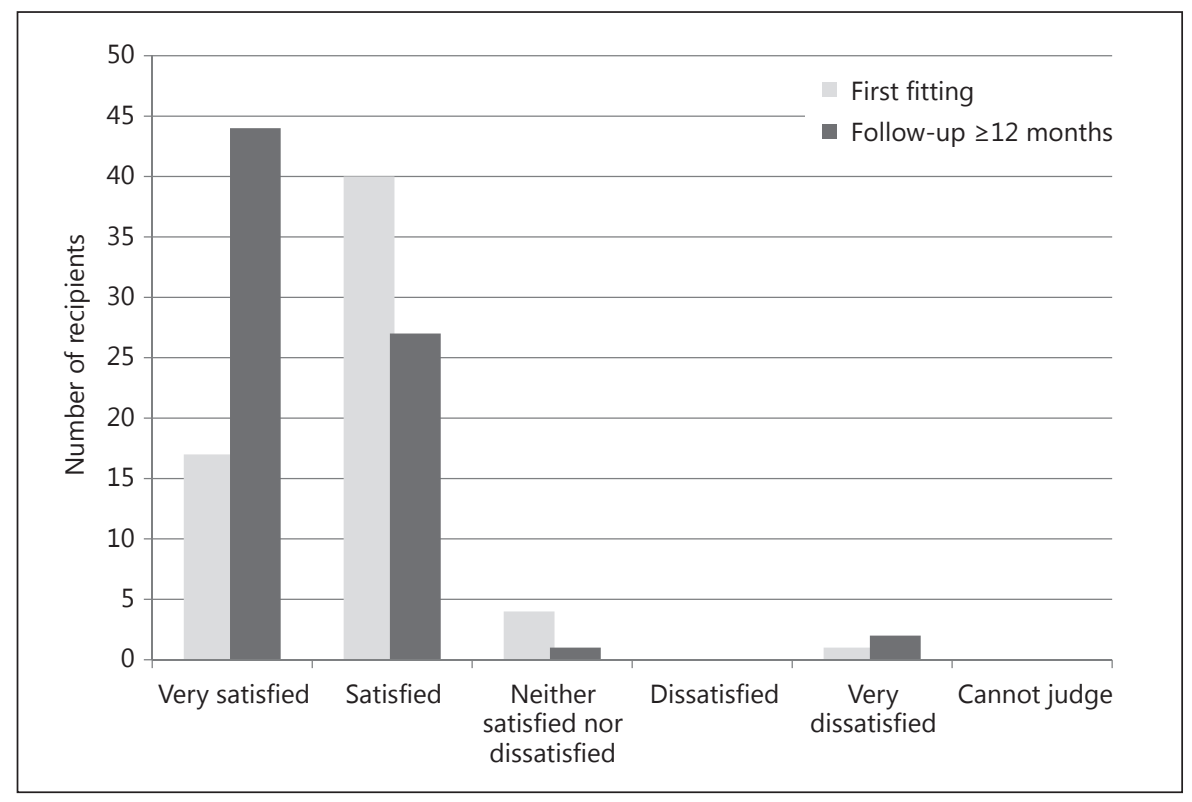

Fig. 2. Reports of patient satisfaction at the first fitting and $\geq 12$ months of follow-up.

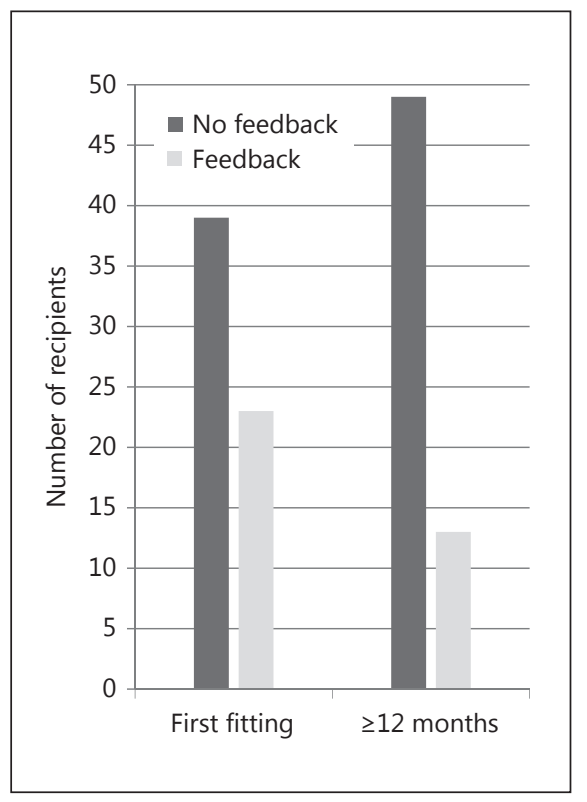

Fig. 3. Reports of feedback at the first fitting and $\geq 12$ months of postimplantation follow-up. these included: at both intervals - gender, contralateral HA use after implantation, and the primary motivation for implantation; at first fitting only - pre-implantation HA use in the implanted ear, hearing loss degree in the contralateral ear and ear canal size; at $\geq 12$ months after fitting only - age at implantation and ear canal direction. Generally speaking, considering longitudinal results and correlations, feedback was more likely associated with females than males, postimplantation contralateral HA use compared to no contralateral HA, and having a primary motivation associated with work requirements for hearing rather than cosmetics or not being able to use an HA. While not consistent over time, "eyeballed categorisation" of ear canal characteristics suggested that those with average-sized ear canals were more likely to experience feedback initially than those with narrow ear canals while those with winding ear canals were more likely to report feedback at $\geq 12$ months than those with straight ones.

\section{Surgical Parameters for Carina Cohort}

The surgical parameters examined and reported for the 62 recipients (66 ears) are shown in Table 3. At the time of review, the majority of recipients presented with the Otologics Carina implant using the T2 transducer and $1 / 5$ used the Cochlear Carina implant released in 2014. While all used the T2 transducer at the follow-up assessment, due to failed legacy versions of the Otologics Carina with a $\mathrm{T} 1$ transducer, 5 cases, originally presenting with a $\mathrm{T} 1$ at the first fitting, had undergone re-implantation with a Cochlear Carina. In reference to Figure 1 , showing positions $\mathrm{A}-\mathrm{E}$ for the implantable microphone, the most frequently used location for the cohort was on the posterior inferior mastoid line (B) in $65 \%$ of cases, followed by mastoid tip (A) in $15 \%$, and posterior mastoid (C) in $11 \%$ of cases. The remaining $9 \%$ were retro-auricular at the occipital level (D). No cases were reported to have the microphone in a posterior superior position (E). The majority of cases, $97 \%$, had 1 or more means of fixation for the microphone to the surrounding bone and/or tissue. A high level of consistency was reported by the co-authors for the coupling location of the transducer to the ossicular chain, being the body of the incus in $97 \%$ of cases, and for the reported absence of any specific preparation of the coupling location in $94 \%$ of cases.

\section{Surgical Variables versus Patient Outcomes}

Statistical analysis examined the correlation of surgical parameters examined and the reported patient outcomes over time with the variables examined listed in Table 3. The level of reported patient satisfaction showed no correlation with any of the surgical parameters examined. 
Table 3. Surgical variables for the study cohort: correlation of variables with reports of feedback

\begin{tabular}{|c|c|c|c|c|c|c|c|c|c|}
\hline \multirow[t]{2}{*}{ Variable } & \multicolumn{2}{|c|}{$\begin{array}{l}\text { Feedback at first } \\
\text { switch-on, } n\end{array}$} & \multirow[t]{2}{*}{$\begin{array}{l}\text { Total, } \\
n\end{array}$} & \multirow[t]{2}{*}{$p$ value } & \multicolumn{2}{|c|}{$\begin{array}{l}\text { Feedback at } 12 \\
\text { months, } n\end{array}$} & \multirow[t]{2}{*}{$\begin{array}{l}\text { Total, } \\
n\end{array}$} & \multirow[t]{2}{*}{$\begin{array}{l}p \\
\text { value }\end{array}$} & \multirow{2}{*}{$\begin{array}{l}\text { Counts } \\
\text { for } 66 \\
\text { ears }\end{array}$} \\
\hline & no & yes & & & no & yes & & & \\
\hline \multicolumn{4}{|l|}{ Microphone location (see Fig. 1) } & \multicolumn{3}{|l|}{$<0.001$} & & \multicolumn{2}{|l|}{0.01} \\
\hline A (mastoid tip) & $1(10)$ & $9(90)$ & $10(16)$ & & $4(40)$ & $6(60)$ & $10(16)$ & & $10(15)$ \\
\hline B (posterior inferior mastoid line) & $27(69)$ & $12(31)$ & $39(63)$ & & $32(82)$ & $7(18)$ & $39(63)$ & & $43(65)$ \\
\hline E (posterior superior) & $0(0)$ & $0(0)$ & $0(0)$ & & $0(0)$ & $0(0)$ & $0(0)$ & & $0(0)$ \\
\hline \multicolumn{4}{|l|}{ Device brand } & \multicolumn{3}{|l|}{0.106} & & \multicolumn{2}{|l|}{0.717} \\
\hline Otologics Carina & $28(57)$ & $21(43)$ & 49 (79) & & $38(78)$ & $11(22)$ & $49(79)$ & & $53(80)$ \\
\hline Cochlear Carina & $11(85)$ & $2(15)$ & $13(21)$ & \multirow{2}{*}{\multicolumn{3}{|c|}{ ) }} & $13(21)$ & & $13(20)$ \\
\hline \multicolumn{4}{|l|}{ Actuator location } & & & & & \multicolumn{2}{|l|}{1} \\
\hline Drill & $1(100)$ & $0(0)$ & $1(2)$ & & $1(100)$ & $0(0)$ & $1(2)$ & & $1(2)$ \\
\hline Laser & $1(100)$ & $0(0)$ & $1(2)$ & & $1(100)$ & $0(0)$ & $1(2)$ & & $1(2)$ \\
\hline None & $35(60)$ & $23(40)$ & $58(97)$ & & $45(78)$ & $13(22)$ & $58(97)$ & & $64(97)$ \\
\hline \multicolumn{4}{|l|}{ Microphone fixation } & \multicolumn{3}{|l|}{0.473} & & \multicolumn{2}{|c|}{0.37} \\
\hline Screws & $17(71)$ & $7(29)$ & $24(39)$ & & $20(83)$ & $4(17)$ & $24(39)$ & & $28(43)$ \\
\hline Sutures & $4(67)$ & $2(33)$ & $6(10)$ & & $6(100)$ & $0(0)$ & $6(10)$ & & $6(9)$ \\
\hline Both & $16(53)$ & $14(47)$ & $30(48)$ & & $21(70)$ & $9(30)$ & $30(48)$ & & $30(46)$ \\
\hline None & $2(100)$ & $0(0)$ & $2(3)$ & & $2(100)$ & $0(0)$ & $2(3)$ & & $2(3)$ \\
\hline \multicolumn{4}{|l|}{ Actuator at first fitting } & \multicolumn{3}{|l|}{0.148} & & \multicolumn{2}{|l|}{0.574} \\
\hline $\mathrm{T} 2$ & $34(60)$ & $23(40)$ & $57(92)$ & & $44(77)$ & $13(23)$ & $57(92)$ & & $61(92)$ \\
\hline $\mathrm{T} 1$ & $5(100)$ & $0(0)$ & $5(8)$ & & $5(100)$ & $0(0)$ & $5(8)$ & & $5(8)$ \\
\hline \multicolumn{4}{|l|}{ Actuator at follow-up } & 1 & & & & 1 & \\
\hline
\end{tabular}

Figures in parentheses are percentages. $p$ values of significance are italicised. T2, current transducer model; T1, predecessor transducer model.

The reported system feedback showed a significant correlation with microphone location at both assessment intervals, being strongest at the first fitting. Feedback was more likely to be reported for recipients with position A, with 90 and $60 \%$ of cases reporting feedback at the first fitting and follow-up assessment, respectively. Thirty percent of cases with position B reported feedback initially, which was reduced to $18 \%$ at the follow-up assessment. Although a small set, no cases with position $\mathrm{D}$ reported feedback while $1 / 3$ of those with position $C$ experienced feedback at the first fitting.

Preferred Surgical Procedure for Microphone Location

Descriptive surgical methods provided by each author are collectively summarised with details provided in the Appendix. Good agreement was arrived at by co-authors for all aspects related to evaluation, identification and preferred location for seating the microphone and its fixation. In summary the preferred location for microphone location is on the posterior inferior mastoid line corresponding to position B in Figure 1, whenever possible. The preferred location independently described is consistent with the most frequently used microphone position in $65 \%$ of cases included in this report and reviewed as shown in Table 3 and Figure 1.

\section{Correlation between Longitudinal Patient Outcomes}

Statistical examination of the relationship between the report of feedback and patient satisfaction was performed and is shown in Table 4 demonstrating the cross-correlation of outcomes at both assessment intervals and their respective $p$ values. A significant negative correlation is 
Table 4. Correlation of patient outcomes for reported satisfaction and system feedback at the first fitting and at $>12$ months of followup $(n=62)$

\begin{tabular}{|c|c|c|c|c|c|c|c|c|}
\hline \multirow[t]{2}{*}{ Variable } & \multicolumn{2}{|c|}{$\begin{array}{l}\text { Feedback at first } \\
\text { fitting, } n\end{array}$} & \multirow[t]{2}{*}{$\begin{array}{l}\text { Total, } \\
n\end{array}$} & \multirow[t]{2}{*}{$p$ value } & \multicolumn{2}{|c|}{$\begin{array}{l}\text { Feedback at } 12 \text { months, } \\
n\end{array}$} & \multirow[t]{2}{*}{$\begin{array}{l}\text { Total, } \\
n\end{array}$} & \multirow[t]{2}{*}{$p$ value } \\
\hline & no & yes & & & no & yes & & \\
\hline Patient satisfaction at first fitting & & & & 0.059 & & & & 0.006 \\
\hline At least dissatisfied & $0(0)$ & $1(100)$ & $1(2)$ & & $0(0)$ & $1(100)$ & $1(2)$ & \\
\hline $50 / 50$ & $1(25)$ & $3(75)$ & $4(6)$ & & $1(25)$ & $3(75)$ & $4(6)$ & \\
\hline At least satisfied & $38(67)$ & $19(23)$ & $57(92)$ & & $48(84)$ & $9(16)$ & $57(92)$ & \\
\hline Patient satisfaction at $>12$ months & & & & 0.047 & & & & 0.008 \\
\hline At least dissatisfied & $0(0)$ & $2(100)$ & $2(3)$ & & $0(0)$ & $2(100)$ & $2(3)$ & \\
\hline $50 / 50$ & $0(0)$ & $1(100)$ & $1(2)$ & & $0(0)$ & $1(100)$ & $1(2)$ & \\
\hline At least satisfied & $39(66)$ & $20(34)$ & $59(95)$ & & $49(83)$ & $10(17)$ & $59(95)$ & \\
\hline
\end{tabular}

Figures in parentheses are percentages. $p$ values of significance are italicised. 50/50, mid-response scale point for patient satisfaction.

observed with reports of feedback at the first fitting and patient satisfaction at $\geq 12$ months after implantation. Reports of feedback at $\geq 12$ months are significantly linked to poorer patient satisfaction ratings at both assessment intervals $(p=0.006-0.008)$. While no recipient without feedback was reported as dissatisfied, a small group, between 16 and $23 \%$, of satisfied recipients also reported experiencing system feedback.

\section{Summary and Discussion}

Data analysis from the recorded experience in a cohort of 62 Carina recipients from 3 of the 5 co-author sites demonstrates that reported patient satisfaction increased over time while reports of system feedback, similar to typical acoustic feedback experienced with conventional amplification, decreased for the cohort. Furthermore, a significant negative correlation was observed between reports of system feedback and the level of patient satisfaction reported at both assessment intervals suggesting patient satisfaction as a function of reported feedback. While no case who did not report system feedback was considered dissatisfied, a small group of cases who reported feedback were considered satisfied.

The cohort represents an experienced adult Carina user group with long-term experience of 3.5 years on average, suggesting that AMEI treatment is a successful long-term alternative when conventional acoustic amplification is not suitable or not desired by the hearing-impaired individual. Consistent daily use was reported for
$97 \%$ of the cohort who wore their system $10 \mathrm{~h} /$ day. Daily use of the device may also be considered another possible indicator of treatment success [Rameh et al., 2010]. The 2 exceptions who used their device for 1-3 h/day only both reported very bothersome feedback, but nonetheless were divergent in their level of satisfaction with one reported as very satisfied while the other was very dissatisfied.

The majority of recipients presented with a significant moderate-to-severe sensorineural hearing loss, and symmetry for interaural hearing loss configuration in most cases. Prior to implant treatment, conventional amplification was used in the majority of cases, 79\% ipsilaterally and $70 \%$ contralaterally. Given that the average age at implantation of the cohort was 39 years, it is perhaps not surprising that the primary motivation for implantation was recorded as work related for more than $52 \%$ of the cases, while $27 \%$ listed cosmetics as a primary motivation. Medical contraindication to wearing a conventional amplification was rare in this group, with aetiologies such as chronic otitis and congenital atresia reported for only $7 \%$ $(4 / 66)$ of the treated ears. Only 1 case was primarily motivated for implant treatment by the inability to use conventional amplification; however, $20 \%$ of the group did not use HAs before implantation although the reasons as to why not are available. In our study, details of the primary motivation for each case were not gathered. Such details may be clinically important, at least on an individual basis, in order to help mould the appropriate expectations before surgery. While patient satisfaction is clearly individual, understanding the motivations including why some patients may prefer bilateral Carina im- 
plants versus a bimodal device (i.e. Carina and a contralateral HA) may impact not only hearing performance outcomes but patient satisfaction.

The most commonly used microphone location for the study cohort was the posterior inferior mastoid line (position B), in $65 \%$ of recipients, followed by the mastoid tip (A) in $15 \%$, the posterior mastoid (C) in $11 \%$, and the retroauricular location at the occipital level (D) in $9 \%$. No microphones were reported in a posterior superior position (E) for this study cohort, although this position had been used by some of the surgeons historically. Position $B$ is consistent with the preferred location described independently by the co-authors and summarised for their collective clinical opinion for the evaluation and selection of the microphone location (Appendix). The authors agree that whenever anatomically feasible, the posterior inferior mastoid line (position B) is desirable, followed by a retroauricular (posterior) site (position $\mathrm{D}$ ). Both locations are nominated taking into consideration the need to provide microphone body fixation opportunities for stability, maximum sound reception through the skin, and to leverage of the potential physical advantages of the pinna in shielding against low levels of acoustic feedback pending individual characteristics.

Examination of various surgical details and patient characteristics gathered for the cohort was undertaken to determine their possible influence upon patient satisfaction and system feedback for clinical consideration. Reports of patient satisfaction for the cohort did not correlate with any of the variables examined at either assessment interval. In contrast, reports of system feedback showed a significant correlation with a small set of patient variables and 1 surgical variable. System feedback reported at $\geq 12$ months was shown to occur more likely in younger recipients, females, those wearing a contralateral HA before and after implantation, recipients with motivations including work and lifestyle needs and individuals with straight versus winding ear canals. As ear canal characteristics were eyeballed only and then characterised for response, not much can be inferred due to possible inherent judgement bias; however, it may suggest a trend for potential influence of physical characteristics of the ear canal upon the likelihood for feedback and may warrant further more rigorous investigation. As females made up $40 \%$ of the cohort and were more likely to experience feedback than males, physical characteristics of the female head may have an influence on feedback and consideration of microphone location.

The significant correlation between motivation for implant treatment and reports of feedback was evidenced in more than half (52\%) of the recipients; those motivated for work-related issues were more likely to experience feedback than those motivated by cosmetic appearance (27\%). Counselling is thus of the utmost importance to determine the motivations, for the implant treatment in one or both ears can be matched to appropriate expectations, and to ensure overall amplification needs in daily dynamic work and home environments can be met technically by the fully implantable system. Furthermore consideration of additional amplification options as hearing needs arise, such as via external amplification, may be important, especially for those with severer degrees of hearing loss.

The correlation between reported system feedback at the first fitting and follow-up assessment, with the use of a contralateral HA before and after implantation, raises questions concerning the possible mechanisms involved in the presence of a contralateral HA. Any disturbance in the physical feedback pathway, such as a change in tissue thickness or the presence of a reflective surface, e.g. a wall close to the patient's head, will change the feedback loop transfer function ("loop gain") and lead to a change in perceived feedback. System feedback in an AMEI may be through direct conduction of vibration through bone and tissue from the vibrating actuator to the microphone, or through acoustic transmission of sound radiated from the tympanic membrane and thus to the microphone, or through both pathways. The presence of a contralateral postimplantation HA may influence the feedback loop transfer function (loop gain) and lead to a change in perceived feedback, passively through its influence on the conductive pathway. Alternatively a contralateral HA may actively influence the loop gain by transmitting acoustic signals that influence the output of the AMEI. The correlation with the report of pre-implantation contralateral HA use and feedback over time is possibly associated with the fact that individuals wearing a contralateral pre-implantation HA are largely the same individuals wearing it after implantation.

Clinical measures may be considered to influence and reduce the potential for the feedback loop mechanism. In some cases, surgeons have found it necessary to surgically shift the location of the implanted microphone to reduce feedback [Bruschini et al., 2010]. Technically it is also possible to make improvements to the feedback reduction filter employed in the Carina system during routine audiological visits in which the individual changes posture systematically while the filter characteristics are adjusted [Uhler et al., 2016]. 
(Note: The feedback reduction filter used in the Carina system compensates for the signal returning to the microphone in the feedback loop, by comparing it to the output stimulation signal and adjusting filter parameters accordingly, and thus the available gain [pers. commun. from technical advisor from Cochlear Boulder, Boulder, CO, USA].)

Clinical practices are evolving as experience grows. Although it is difficult to compare definitively, it is likely that the most frequent microphone location used in our study, the posterior inferior mastoid line (position B), is consistent with that most used and described as "close to the mastoid tip" by Bruschini et al. [2016]. As mentioned above, their earlier work confirmed the need to consider microphone location and its impact on the feedback loop with possible repositioning when not optimal for the individual [Bruschini et al., 2010]. While the earlier published manufacturer recommendation for location of the microphone suggested the retro-auricular posterior position, similar to that described as posterior auricular (position D), this was used in only $9 \%$ of our study cohort [Martin et al., 2009]. A small mastoid mass is one of the factors affecting the surgeon's recommendation to utilise this placement in some cases. In our study cohort, the mastoid tip (position A) used in 15\% of cases was the only location linked to an increased likelihood for feedback. The potential reasons for an increased likelihood of feedback reports in this position may be manifold and possibly include reduced shielding effects of the pinna and a closer proximity to the ear canal relative to when the posterior inferior mastoid line or retro-auricular positions are employed.

Complications were reported as low for the study cohort. Two cases of microphone body extrusion were reported that were addressed by minor surgical revision. This highlights the importance of ensuring that the skin flap over the microphone body is sufficiently thick to prevent tissue necrosis, although this complication is relatively rare [Jenkins et al., 2008], while not exceeding the recommended $6 \mathrm{~mm}$ to avoid any significant attenuation of the microphone reception [Jenkins et al., 2007].

Five cases in our study cohort, originally implanted with an Otologics Carina T1 device, underwent re-implantation due to technical failure; 4 were re-implanted with an Otologics Carina T2 device prior to 2014 and 1 with a Cochlear Carina in 2014. All were re-implanted in the same ear and are satisfied, all-day users. No major complications were reported following the long-term use of the Otologics Carina T2 device or the Cochlear Carina in these cases.
As reported previously, successive generations of the Otologics Carina transducer have demonstrated an increased reliability compared to earlier generations [Jenkins and Uhler, 2014; Debeaupte et al., 2015]. Our data confirm the reliability of the Cochlear Carina, in the subset of 15 ears, following 2 years of user experience at the time of publication. It should be noted, as shown in Table 2, that there was no correlation with patient satisfaction and device brand when incorporating the T2 transducer.

Patient satisfaction and patient reports of feedback were given in this study via a patient file examination and individual review of retrospectively treated Carina recipients. While this retrospective study provides a good overview of the current status and outcomes for a moderate-sized cohort of Carina users, an inherent bias is introduced by recall and proxy judgement of patient progress and is dependent on the consistency of historical records. For future studies, whether retrospective or prospective, with the aim to review treatment outcomes for implanted cohorts of hearing-impaired individuals, the implementation of consistent methods for clinical evaluation and data record will be beneficial for data analysis and its interpretation, and ultimately clinical application.

\section{Conclusion}

The majority of the 62 hearing-impaired adults in our multicentre study cohort, using the Carina system with the T2 actuator, are reported as satisfied, consistent allday users, with experience of 3.5 years on average. Patient satisfaction is shown to increase over time, and reports of feedback decrease over time. While no patient variables or surgical variables were linked to patient satisfaction, the microphone location is shown to have a significant influence on feedback reports. As patient satisfaction is influenced by reports of system feedback, it is important to minimise the potential for feedback by appropriate patient counselling, patient selection, and surgical procedures.

The most frequently reported implantable microphone location for the study cohort was the posterior inferior mastoid line which is consistent with the preferred location described by the authors whenever anatomically possible. This location as well as other posterior locations are less likely to be associated with reports of system feedback compared to a mastoid tip position. 


\section{Appendix}

The surgical experience described in this Appendix is a reflection of the clinical experience independently reported by each author, which has been consolidated with their collaborative agreement to provide their collective clinical opinion based on over 160 Carina surgeries between them. The surgical steps described are provided as their agreed and preferred method to identify the location for the implantable microphone of the Carina system on a case-by-case basis. Their opinion is not a reflection of the experience of all surgeons familiar with and providing equivalent hearing implant treatments on a routine basis to hearing-impaired individuals. A review of the related literature as presented in this paper revealed very little detail on the evaluation procedures, surgical steps and considerations involved in determining the implantable microphone location in clinical practice on a case-by-case basis. Hence, the information provided in this Appendix is a detailed description of the "How I do it" by the surgeons and authors of this paper.

\section{Overview of Surgical Guidelines}

The 5 authors of this paper, all implant surgeons, with over 160 Carina surgeries between them including earlier legacy systems of the currently available Carina system, provided their individual and independent recommendations for the most important steps to evaluate, identify the site, and secure the implantable microphone. The summary of their individual contributions is provided as their consolidated opinion based on their collective clinical experiences. The primary steps to consider for selecting the microphone location are consistent across the author group. The preferred location for the implantable microphone, whenever anatomically possible, is a posterior inferior mastoid line, followed by a posterior retro-auricular position. The following represents the co-author consolidated guidelines.

General Considerations. As a first step, assess the general appearance of a patient's physical characteristics such as the length of the neck, skin thickness and condition of the skin in the surrounding retro-auricular area, including any anomalies in skin attachment and mastoid bone dimensions that may prevent placement and fixation of the microphone body. Similarly, evidence of previous surgeries and any residual scarring should be taken into account when determining the microphone location (e.g., mastoidectomy, multiple tympanoplasty or aural atresia surgery).

Audiological Assessment. Pure-tone audiometry is required to identify the amplification needs for the potential implant ear(s). Higher gain requirements may increase the likelihood of feedback with less risk usually observed for conductive or mixed hearing loss cases than for those with sensorineural hearing loss. Consistent with product labelling, the targeted hearing loss upper limit is a moderate-to-severe degree of hearing loss for the average bone conduction thresholds across the frequency range. In addition, speech audiometry needs to be performed to ensure sufficient baseline speech intelligibility. Furthermore, a trial with a conventional HA(s) is recommended to predict the required amplification to improve audibility and to provide the patient with an idea of the achievable results.

Radiological Assessment. Pre-operative imaging (computerised tomography or cone beam computer tomography of the temporal bone) is recommended to determine optimal positions for all Carina implant components. A computer tomography scan is essen- tial prior to surgery to evaluate the physical characteristics of the bone mass in the desired area targeted for implantation, the retrosigmoid mastoid bone, superior to the temporal line. Any potential difficulties in seating the individual components of the Carina implant system, consisting of the actuator, implant body and microphone body, are assessed in relation to the overall skull shape and thickness, the proximity of the meninges, otic capsule and mastoid bone. While generally not problematic, it is important that exposure of the dura and the lateral and transverse sinuses is minimised and, at best, avoided during creation of the implant body and microphone body bone beds to prevent any resulting injury including cerebral spinal fluid leakage, bleeding or secondary thrombophlebitis. Furthermore, exposure and contact of the microphone with the vascular structures, such as the sigmoid sinus, should be avoided to prevent the perception of vascular flow noise that, in turn, may impact the effectiveness of the noise cancellation algorithm when used to reduce perception of body noises in general.

Physical Examination. Palpation of the retro-auricular area using the fingertip is recommended to assess the location of the inferior posterior mastoid bone, the mastoid bone process, the thickness of the skin, and the subcutaneous cell tissue. Otomicroscopy is performed to evaluate the physical attributes and condition of the ear canal, including its diameter, morphology on the anteriorposterior and superior-inferior planes, and the curvature.

Identification of the Microphone Position. Presurgical determination of the potential microphone placement is possible in the majority of cases. The preferred microphone location is on the mastoid bone process whenever anatomically possible. In general, the retrosigmoid/mastoid process bone area is easily accessible, has sufficient bone thickness, is ideally placed to adequately receive external sounds, in particular conversational speech, and to limit reception and amplification of internal body noises.

Microphone Placement. The aim is for the microphone body to be seated on the mastoid process, in a posterior-inferior position, whenever possible. In this position, the pinna acts as an effective barrier against sound emitted from the ear canal reaching the microphone, which can help to avoid feedback.

(Note: For all locations, ideally the skin layer over the microphone membrane is kept thin, no more than $6 \mathrm{~mm}$, to optimise reception of external sounds.)

Care must be taken to ensure the microphone is not in contact with the system hardware, such as the connecting cable, implant body, and mounting bracket, or with internal anatomical structures, vascular and muscular, to avoid possible reception of body noises, possible movement and subsequent feedback.

(Note: For all locations, the complete absence of any contact between the mounting bracket and the microphone is important.)

\section{Considerations for a Mastoid Tip Location}

(a) The microphone body should be placed at the level of the mastoid process, ideally in a posterior-inferior position, whenever anatomically possible. Using a microphone template, a bone bed is drilled on the mastoid process to seat the microphone body. Pending the size and convexity of the mastoid bone process, the bed may be an incomplete circle.

(b) The microphone body should not protrude beyond the cortical plane in order to avoid pressure on the pinna while sleeping or leaning the head on a firm surface that may lead to physical discomfort. 
(c) Seated inferior-posterior to the mounting bracket, the cable from the implant body should enter the microphone body with a descending oblique direction posteriorly. Care is taken to avoid contact between the final position of the cable and microphone membrane.

(d) Fixation of both titanium wings of the microphone body to the temporal bone is recommended to prevent undesired movement and contact. In the posterior-inferior position, a screw through the anterior wing seated over bone and a silk suture through the posterior wing may be used if seated over tissue. However, if seated entirely on bone, although rare in view of the size of the mastoid bone needed, 2 screws may be used. A single point of fixation is not recommended in order to avoid unnecessary movement and a potential increased risk of feedback.

(e) The angle of the wings may be adjusted as needed to avoid any contact with other components of the implant, including the mounting bracket. To further accommodate the microphone body in the desired location, the mounting bracket inferior arms may be shortened (subsequently leaving a smaller number of screws to secure the mounting bracket).

(f) The skin flap over the microphone membrane should be ideally hair free and a well-vascularised subcutaneous layer of tissue between 3 and $6 \mathrm{~mm}$ thick.

Special Considerations in Select Cases for Subcutaneous Pocket

Placement

There are several reasons why a mastoid process location may not possible. Issues that may prevent a mastoid process location and thus require a subcutaneous pocket may include any of the following: a small mastoid mass; thick skin (fatty or fibrous tissue) covering the mastoid area, usually seen in males; previous large otological surgical scarring; anomalies in hair attachment, and lifestyle issues with the need to accommodate proximity to articles of clothing and or accessories worn daily or during sport.

In these cases, another option for the microphone location is retro-auricular at the occipital height. In this position, attachment to the cortex of the occipital bone with screws depending on the thickness of the skin is recommended. Similarly to the posterior inferior mastoid line position, the pinna may act as a physical barrier to sound emitted from the ear canal and may help to reduce feedback.

Alternatively, a subcutaneous pocket may be created posterior to the sternocleidomastoid muscle and as superior as possible in the neck to seat the microphone. This position may reduce the potential for reception of the patient's voice relative to a more inferior position on the neck. In this position, the pinna will likely have less effect as a physical barrier to the sound emitted from the ear canal than when placed on the mastoid bone or in the retro-auricular position at the occipital height. To secure the implant, silk thread may be used through the microphone body wings within the subcutaneous pocket. As far as possible, flat positioning of the microphone body is desired, avoiding any irregular angulation or bulging which may create areas of tissue susceptible to irritation.

The authors hypothesise that a subcutaneous pocket may reduce the potential for vibratory energy to be transferred from the actuator to the microphone via the fixation screws in contact with the bone. However, noises related to textiles such as scarves, ties and other clothing may be significantly amplified and thus a neck position is rarely recommended.

(Note: For all locations, special attention to the location of the incision line is needed.)

The incision line should always be kept away from the final position of the microphone membrane. Keloid scarring along the incision line can greatly disrupt the fitting of the implant system given the high level of cellular activity, which can change the acoustic impedance of the tissue that covers the microphone's membrane. Furthermore, if the microphone is situated directly beneath the incision line, the chances for extrusion and compromised vascularisation of the area are increased.

\section{Acknowledgements}

We would like to thank Dr. Petra Graham at the Macquarie University, Sydney, Australia, for her expert advice and statistical analysis in the examination of the surveyed data for the Carina recipient cohort as a consultant statistical advisor to Cochlear AG in Basel, Switzerland. We would like to sincerely thank the clinical team members for their support in the evaluation and data collection for the Carina recipients included in the analysis. In particular, we would like to thank Alessandra Murri in Italy, Paul Berger and Yannick Dumouchel in France, and Marta Cortijo Torres in Spain.

\section{Statement of Ethics}

Evaluations for this report were performed during routine clinical management of Carina recipients in each clinic. All clinical assessments were conducted according to the principles expressed in the Declaration of Helsinki for the protection of human rights and patient data privacy.

\section{Disclosure Statement}

The authors declare that there is no conflict of interest or funding for participation in this study.

\section{References}

Bittencourt A, Burke P, Jardim Ide S, Brito R, Tsuji R, Fonseca A, Bento R: Implantable and semi-implantable hearing aids: a review of history, indications, and surgery. Int Arch Otorhinolaryngol 2014;18:303-310.

Bruschini L, Berrettini S, Forli F, Muri A, Cuda D: The Carina ${ }^{\odot}$ middle ear implant: surgical and functional outcomes. Eur Arch Otorhinolaryngol 2016;273:3631-3640.

Bruschini L, Forli F, Passetti S, Bruschini P, Berrettini S: Fully implantable Otologics MET Carina device for the treatment of sensorineural and mixed hearing loss: audio-otological results. Acta Otolaryngol 2010;130:1147-1153. 
Debeaupte M, Decullier E, Tringali S, Devèze A, Mom T, Darrouzet V, Truy E: Evolution of the reliability of the fully implantable middle ear transducer over successive generations. Otol Neurotol 2015;36:625-630.

Egolf D, Haley B, Howell H, Legowski S, Larson $\mathrm{V}$ : Simulating the open-loop transfer function as a means for understanding acoustic feedback in hearing aids. J Acoust Soc Am 1989; 85:454-467.

Haynes D, Young J, Wanna G, Glasscock M: Middle ear implantable hearing devices: an overview. Trends Amplif 2009;13:206-214.

Jenkins H, Atkins J, Horlbeck D, Hoffer M, Balough B, Alexiades G, Garvis W: Otologics fully implantable hearing system: phase I trial 1-year results. Otol Neurotol 2008;29:534541.

Jenkins H, Atkins J, Horlbeck D, Hoffer M, Balough B, Arigo J, Alexiades G, Garvis W: US phase I preliminary results of use of the Otologics MET fully implantable ossicular stimulator. OtolaryngoI Hlead Neck Surg 2007; 137: 206-212.

Jenkins $\mathrm{H}$, Uhler $\mathrm{K}$ : Otologics active middle ear implants. Otolaryngol Clin North Am 2014; 47:967-978.
Kam A, Sung J, Yu J, Tong M, de Abajo J, Sanhueza I, Giron L, Manrique M: Clinical evaluation of a fully implantable hearing device in six patients with mixed and sensorineural hearing loss: our experience. Otol Neurotol 2013;34:1373-1379.

Lefebvre P, Martin C, Dubreuil C, Decat M, Yazbeck A, Kasic J, Tringali S: A pilot study of the safety and performance of the Otologics fully implantable hearing device: transducing sounds via the round window membrane to the inner ear. Audiol Neurotol 2009;14:172180.

Martin C, Deveze A, Richard C, Lefebvre P, Decat M, Ibañez L, Truy E, Mom T, Lavieille J, Magnan J, Dubreuil C, Tringali S: European results with totally implantable Carina placed on the round window: 2-year follow-up. Otol Neurotol 2009;30:1196-1203.

Pulcherio J, Bittencourt A, Burke P, Monsanto R da C, de Brito R, Tsuji R, Bento R: Carina ${ }^{\circledR}$ and Esteem ${ }^{\circledR}$ : a systematic review of fully implantable hearing devices. PLoS One 2014; 9:e110636.

Rameh C, Meller R, Lavieille JP, Deveze A, Magnan J: Long-term patient satisfaction with different middle ear hearing implants in sensorineural hearing loss. Otology \& Neurotology 2010;31:883-892.
R Core Team: R: a language and environment for statistical computing. R Foundation for Statistical Computing, Vienna, 2016. https:// www.R-project.org/.

Savaş V, Gündüz B, Karamert R, Cevizci R, Düzlü M, Tutar H, Bayazit Y: Comparison of Carina active middle-ear implant with conventional hearing aids for mixed hearing loss. J Laryngol Otol 2016;130:340-343.

Uhler K, Anderson C, Jenkins H: Long-term outcome data in patients following one year's use of a fully implantable active middle ear implant. Audiol Neurotol 2016;21:105-112.

Zenner HP, Leysieffer H: Total implantation of the Implex TICA hearing amplifier implant for high frequency sensorineural hearing loss: the Tübingen University experience. Otolaryngol Clin North Am 2001;34:417-446.

Zwartenkot JW, Hashemi J, Cremers CW, Mulder JJ, Snik AF: Active middle ear implantation for patients with sensorineural hearing loss and external otitis: long-term outcome in patient satisfaction. Otol Neurotol 2013;34:855861 